\title{
Association of Oral Function With Long-Term Participation in Community-Based Oral Exercise Programs in Older Japanese Women: A Cross-Sectional Study
}

\author{
Sanae Miyoshia, b, Hideo Shigeishia, d, Eri Fukada ${ }^{\text {a }}$, Maki Nosou ${ }^{a}$, \\ Hideaki Amano ${ }^{\mathrm{c}}$, Masaru Sugiyama ${ }^{\mathrm{a}}$
}

\begin{abstract}
Background: There are several researches demonstrating that community-based educational and exercise programs can improve oral function in older people. However, the relationship between oral function and long-term participation in health programs has not been fully elucidated. The objective of this study was to clarify the oral health status and oral function of older people ( $\geq 65$ years) who had been participating in community-based exercise programs at community salons.
\end{abstract}

Methods: We enrolled 108 women (mean age: $77.6 \pm 5.7$ years) who participated in oral and physical exercise programs once a week at community salons in Takehara throughout May 2017. This crosssectional pilot study was approved by the Ethical Committee of Hiroshima University and informed consent was obtained from all participants. To assess oral function, the following tests were performed: tongue pressure test, oral diadochokinesis (ODK) measurement, repetitive saliva swallowing test (RSST) and oral wetness measurement.

Results: A significant negative correlation was found between age and ODK or RSST (Spearman's rank correlation; $\mathrm{P}=0.007$ and $\mathrm{P}=$ 0.01 , respectively). The duration of participation ranged widely from 1 month to 7 years (median: 2.3 years). Although there was no significant difference between participation period and oral function, individuals who had been participating for 3 years or longer did not demonstrate a negative relationship between age and oral wetness. Furthermore, they exhibited a weaker negative relationship between age and oral function (i.e. tongue pressure, ODK and RSST) when compared with

Manuscript submitted October 19, 2018, accepted October 31, 2018

aDepartment of Public Oral Health, Program of Oral Health Sciences, Graduate School of Biomedical \& Health Sciences, Hiroshima University, Hiroshima, Japan

'Takehara-Toyota Dental Hygienists' Association, Hiroshima, Japan 'Department of Maxillofacial Functional Development, Program of Oral Health Sciences, Graduate School of Biomedical \& Health Sciences, Hiroshima University, Japan

${ }^{\mathrm{d} C o r r e s p o n d i n g ~ A u t h o r: ~ H i d e o ~ S h i g e i s h i, ~ D e p a r t m e n t ~ o f ~ P u b l i c ~ O r a l ~ H e a l t h, ~}$ Program of Oral Health Sciences, Graduate School of Biomedical \& Health Sciences, Hiroshima University, 1-2-3 Kasumi, Minami-ku, Hiroshima 734 8553, Japan. Email: shige@hiroshima-u.ac.jp

doi: https://doi.org/10.14740/jocmr3664 individuals who had been participating for less than 3 years.

Conclusions: Our results suggest that long-term participation in community salon exercise programs may suppress the deterioration of oral function in older people. Further study will be necessary to clarify the significant correlation between oral function and community-based social activities such as oral exercise.

Keywords: Older people; Oral function; Community-based exercise program

\section{Introduction}

Since Japan is aging more rapidly than any other country in the world, preventive health care becomes more important to prolong healthy life expectancy. The Japanese Ministry of Health, Labor and Welfare has attempted to establish a CommunityBased Integrated Care System Model [1]. In this model, community-based older people can live the rest of their lives in familiar environment, even if they come to need long-term care. It is thought that older people could maintain physical and cognitive function through the promotion of long-term preventive care to avoid health problems [2]. For the preventive health care of older people, it is important to increase their activity in daily life and encourage participation in social activities.

Among community-dwelling older people, social and physical activity at community salons that are located within walking distance is now recommended to facilitate healthy aging by maintaining their functional capacity. Therefore, specialists in rehabilitation (e.g. physical therapists, occupational therapists, speech-language-hearing therapists, public health nurses, dietitians and dental hygienists) play a significant role in providing preventive health care to older people. Recently, dental hygienists have become involved in community dental health services, particularly for older people in Japan.

There are several research papers demonstrating that community-based educational and exercise programs can improve oral function in older people [3-5]. However, the relationship between oral function and long-term participation in health programs has not been fully elucidated. Therefore, as a preliminary assessment, we performed a cross-sectional study to investigate the relationship between duration of participation, 
oral function and oral health status in older people who had been participating in community-based exercise programs in Takehara, Hiroshima.

\section{Materials and Methods}

\section{Subjects}

We included 153 women aged $\geq 65$ years who participated in oral and physical exercise programs once a week across eight community salons in Takehara, Japan throughout May 2017. Since there were only a very small number of male participants at the community salons, we decided to exclude men in this study. Informed consent was obtained from 112 participants. Finally, we enrolled 108 (mean age: $77.6 \pm 5.7$ years) of 112 women after exclusion of four individuals because of their incomplete questionnaire. The study design was approved by the Ethical Committee of Hiroshima University (No. E-741) and all participants signed an informed consent agreement. None of the community-dwelling older people included in this study had been certified as requiring long-term care or support. In addition, they had been participating in both oral and physical exercise programs every week. Oral exercise instruction was provided by a dental hygienist. Oral exercise was performed according to the training method recommended by the Japanese Society of Dysphagia Rehabilitation, which is composed of several swallowing exercises (i.e. pursed-lip breathing, neck rotation exercise, shoulder-up and -down exercises, arm exercise, ballooning and pursing exercise of the cheeks, tongue exercise, salivary gland massage and pronunciation practice) [6]. To assess oral status, a dental hygienist examined the teeth present, excluding retained roots, and the use of dentures. Next, oral bacterial counts, oral wetness measurement and oral function tests were performed. Eating, drinking and oral cleaning were prohibited within $30 \mathrm{~min}$ of examination.

\section{Oral bacterial count}

The number of oral bacteria in patient samples was determined using a Bacterial Counter (DU-AA01 NP-H; Panasonic Healthcare Co., Ltd, Tokyo, Japan), with the dielectrophoretic impedance change being converted to bacterial concentration per $\mathrm{mL}$ for each sample (colony-forming units (CFUs)/ $\mathrm{mL}$ ). This device ensures that only viable bacteria are counted. Samples were obtained from the tongue dorsum using a cotton swab, according to the manufacturer's protocols. The number of bacteria was defined as follows: level 1 (lower than $10^{5}$ $\mathrm{CFU} / \mathrm{mL}$ ), level 2 ( $\geq 10^{5}$ to $<10^{6} \mathrm{CFU} / \mathrm{mL}$ ), level $3\left(\geq 10^{6}\right.$ to $<10^{6.5} \mathrm{CFU} / \mathrm{mL}$ ), level 4 ( $\geq 10^{6.5}$ to $\left.<10^{7.0} \mathrm{CFU} / \mathrm{mL}\right)$, level $5\left(\geq 10^{7.0}\right.$ to $\left.<10^{7.5} \mathrm{CFU} / \mathrm{mL}\right)$, level $6\left(\geq 10^{7.5}\right.$ to $<10^{8.0} \mathrm{CFU} /$ $\mathrm{mL})$ and level $7\left(\geq 10^{8.0} \mathrm{CFU} / \mathrm{mL}\right)[7]$.

\section{Oral wetness}

Oral wetness was evaluated using an oral moisture-checking device (Moisture Checker Mucus ${ }^{\circledR}$, Scalar, Tokyo) according to a previous method [8]. The level of oral moisture was measured at the tongue dorsum $10 \mathrm{~mm}$ from the apex linguae. The median values of the measurements were calculated from three independent measurements. A value of $\geq 29.6$ was defined as a normal moisture level.

\section{Tongue pressure}

Maximum tongue pressure was measured using a tongue pressure manometer with a balloon probe (TPM-01; JMS Co. Ltd, Hiroshima, Japan). Measurement was performed according to a previous report [9]. Briefly, the participants were asked to place the balloon probe between the tongue and the anterior part of the palate with the lips closed. The participants were then asked to press the balloon onto the palate for $5 \mathrm{~s}$. The mean value was obtained from three independent measurements.

\section{Oral diadochokinesis}

Oral diadochokinesis (ODK) was used for the evaluation of motor speech abilities. Participants were instructed to repeat the monosyllables $/ \mathrm{pa} /, / \mathrm{ta} /$ and $/ \mathrm{ka} /$ as quickly as possible for $5 \mathrm{~s}$. The number of repetitions was automatically measured by a counting device (Kenko-kun; Takei Scientific Instruments, Niigata, Japan).

\section{Repetitive saliva swallowing test (RSST)}

The participants were instructed to swallow saliva as many times as possible over a 30 -s period. Elevation of the thyroid cartilage during swallowing was assessed by touch. Fewer than three swallows over $30 \mathrm{~s}$ was considered a swallowing disorder.

\section{Data collection using a questionnaire}

To examine the risk of dysphagia and frailty, we conducted a questionnaire investigation. The risk of dysphagia was evaluated using the dysphagia risk assessment for the communitydwelling elderly (DRACE) [10]. This is a reliable method to evaluate the risk of dysphagia in older people and includes 12 evaluation items (i.e. fever, a long time to eat, difficulties with swallowing, difficulties with eating hard food, dropping food from the mouth, choking while eating food, choking while drinking liquid, food entering the nasal cavity after swallowing, voice change after eating food, sensation of food stuck in the esophagus, phlegm at the back of the throat, and sensation of food rising into the mouth from the stomach). The answers include often (score $=2)$, sometimes (score $=1)$ and never (score $=0$ ). According to a previous study [10], we calculated the total score for each given category and considered a DRACE score $\geq 4$ as indicating a high risk of dysphagia.

We also assessed the frailty of the participants using a Ki- 
hon Checklist (KCL) developed by the Japanese Ministry of Health, Labor and Welfare [11]. This checklist is composed of 25 items and several categories (i.e. physical strength, nutritional status, oral function, houseboundness, cognitive status and depression risk). The total of all scores ranged from 0 to 25 . Individuals with a score $\geq 7$ were determined to be in a state of frailty, based on the findings of a previous study [12]. In addition, we asked about the history of dental examination and tooth brushing instruction within the past year and the type of tooth cleaning apparatus regularly used.

\section{Statistical analysis}

Spearman's rank correlation coefficient was used for statistical analysis. The Mann-Whitney $U$ test was used as a nonparametric alternative to the independent $t$-test to assess significant differences, with $\mathrm{P}$ values less than 0.05 regarded to be statistically significant. One-way analysis of variance (ANOVA) with the Tukey post hoc test or Kruskal-Wallis test were also used. Statistical analysis was performed using JMP ${ }^{\circledR}$ Pro 12 software (SAS Institute Inc., Cary, NC, USA).

\section{Results}

\section{Clinical factors of study population}

The participants' clinical factors are summarized in Table 1. The mean number of remaining teeth was $16.8 \pm 9.9$ in 108 participants. Seventy of $108(64.8 \%)$ participants used full (n $=11)$ or partial dentures $(n=59)$. More than $50 \%$ of participants showed a high oral bacterial count (levels 5 and 6 ). The mean DRACE score was $4.3 \pm 3.4$, and 60 (55.6\%) of 108 participants exhibited a DRACE score $\geq 4$, indicating that more than half of the individuals had a risk of dysphagia. The mean KCL score was $5.4 \pm 3.8$. The number of participants with a KCL score $\geq 7$ was 39 (36.1\%). The mean oral moisture value was $30.4 \pm 2.3$. The mean tongue pressure was $30.5 \pm 8.6 \mathrm{kPa}$. Repetition of the monosyllables $/ \mathrm{pa} /, \mathrm{/ta} /$ and $/ \mathrm{ka} /$ occurred 5.7 $\pm 1.0,5.6 \pm 1.0$ and $5.4 \pm 0.9$ times per second, respectively. In addition, the mean RSST value was $3.4 \pm 1.2$ times over $30 \mathrm{~s}$.

The percentage of participants who choked during a meal once a month, several times a week or every day was $13.9 \%$ $(n=15), 5.5 \%(n=6)$ and $0.93 \%(n=1)$, respectively. In addition, the percentage of participants who had received a dental examination or tooth brushing instruction within the past year was $46.2 \%(n=50)$ and $24.0 \%(n=26)$, respectively. Regarding the tooth cleaning apparatus used, the percentage of participants who used a toothbrush alone, a toothbrush and an interdental brush, and a toothbrush, an interdental brush and dental floss was $42.3 \%(\mathrm{n}=44), 42.3 \%(\mathrm{n}=44)$ and $5.8 \%(\mathrm{n}$ $=6$ ), respectively.

\section{Correlation between age and clinical factors}

We examined the correlation between age and clinical factors.
Table 1. Clinical Factors of Study Population

\begin{tabular}{|c|c|}
\hline Clinical factors & $\mathrm{n}(\%)$ \\
\hline \multicolumn{2}{|l|}{ Age in years (mean: $77.6 \pm 5.7$ ) } \\
\hline $65-69$ & $7(6.5 \%)$ \\
\hline $70-79$ & $64(59.3 \%)$ \\
\hline $80-89$ & $36(33.3 \%)$ \\
\hline$\geq 90$ & $1(0.9 \%)$ \\
\hline \multicolumn{2}{|c|}{ Number of remaining teeth (mean: $16.8 \pm 9.9$ ) } \\
\hline$\geq 20$ & $55(50.9 \%)$ \\
\hline $10-19$ & $22(20.4 \%)$ \\
\hline $0-9$ & $31(28.7 \%)$ \\
\hline \multicolumn{2}{|l|}{ Denture } \\
\hline Non-user & $38(35.2 \%)$ \\
\hline Partial denture & $59(54.6 \%)$ \\
\hline Full denture & $11(10.2 \%)$ \\
\hline \multicolumn{2}{|l|}{ Level of bacterial count } \\
\hline Level 2 & $4(3.7 \%)$ \\
\hline Level 3 & $8(7.4 \%)$ \\
\hline Level 4 & $30(27.8 \%)$ \\
\hline Level 5 & $54(50.0 \%)$ \\
\hline Level 6 & $12(11.1 \%)$ \\
\hline \multicolumn{2}{|l|}{ DRACE score (mean: $4.3 \pm 3.4$ ) } \\
\hline$<4$ (low risk of dysphagia) & $48(44.4 \%)$ \\
\hline$\geq 4$ (high risk of dysphagia) & $60(55.6 \%)$ \\
\hline \multicolumn{2}{|l|}{ KCL score (mean: $5.4 \pm 3.8$ ) } \\
\hline$<7$ (non-frailty) & $69(63.9 \%)$ \\
\hline$\geq 7$ (frailty) & $39(36.1 \%)$ \\
\hline
\end{tabular}

There was a significant negative relationship between age and tongue pressure or RSST (Spearman's rank correlation; $r=$ $-0.35, \mathrm{P}<0.001$, and $\mathrm{r}=-0.32, \mathrm{P}=0.01$, respectively). A significant negative correlation was also found between age and ODK (Spearman's rank correlation; $\mathrm{Pa} / \mathrm{r}=-0.21, \mathrm{P}=0.029$; $\mathrm{Ta} / \mathrm{r}=-0.21, \mathrm{P}=0.027$; and $\mathrm{Ka} / \mathrm{r}=-0.26, \mathrm{P}=0.007$, respectively). Furthermore, a significant correlation was found between age and DRACE score (Spearman's rank correlation; $r$ $=0.343, \mathrm{P}=0.011$ ).

\section{Correlation between frailty and clinical factors}

The association of KCL score with oral function parameters such as tongue pressure, ODK, RSST and DRACE was examined. A significant negative association was found between KCL score and ODK (Spearman's rank correlation; $\mathrm{Pa} / \mathrm{r}=$ $-0.28, \mathrm{P}=0.003 ; \mathrm{Ta} / \mathrm{r}=-0.28, \mathrm{P}=0.003 ;$ and $\mathrm{Ka} / \mathrm{r}=-0.36, \mathrm{P}<$ 0.001 , respectively). There was a significant negative association between KCL score and RSST (Spearman's rank correlation; $\mathrm{r}=-0.23, \mathrm{P}=0.019)$. However, no significant correlation was found between KCL score and tongue pressure (Spear- 
man's rank correlation; $\mathrm{r}=-0.12, \mathrm{P}=0.23$ ).

\section{Association of participation period with clinical factors}

The duration of participation ranged widely from 1 month to 7 years (median: 2.3 years). To clarify the effect of the participation period on oral function in older people, we first investigated the correlation between participation duration, oral hygiene status (i.e. moisture level and oral bacterial count) and parameters of oral function (i.e. tongue pressure and ODK) using Spearman's rank correlation coefficient. However, there was no significant difference between participation period and oral hygiene status or oral function. Next, we examined the correlation between participation period by year (i.e. firstseventh) and clinical factors using one-way ANOVA with the Tukey post hoc test or Kruskal-Wallis test (Table 2). However, there was no significant difference between participation period by year and clinical status. In addition, we compared the parameters of oral function between people who had participated for 3 years or longer ( $\geq 3$ years participation group) and those who had participated for less than 3 years $(<3$ years participation group) (Table 3). Statistical analysis was performed using the $t$-test or Mann-Whitney $\mathrm{U}$ test. However, no significant difference was found in the parameters of oral function between the two groups. Furthermore, we examined the correlation between age and parameters of oral function in those two groups. The $\geq 3$ years participation group showed a significant negative relationship between age and the number of remaining teeth (Spearman's rank correlation; $\mathrm{r}=-0.63, \mathrm{P}<$ 0.01 ) (Table 4). Interestingly, the $\geq 3$ years participation group showed a positive relationship between age and oral wetness (Spearman's rank correlation; $r=0.19$ ), while the $<3$ years participation group showed a negative relationship (Table 4). Furthermore, the former group exhibited a much weaker negative relationship between age and oral function when compared with the latter group (Table 4). Conversely, people who had been participating for less than 3 years showed a significant negative correlation between age and oral functions such as tongue pressure, ODK and RSST.

\section{Discussion}

Aging-dependent deterioration has been reported in regards to tongue pressure and ODK in older people $[13,14]$. The deterioration of oral function is attributed to a reduction in masseter muscle volume and poorer masseter muscle and labial movement $[13,14]$. In this study, mean values of oral function in independent older people were similar to the previous results reported by another group in Japan [14]. We also found that age was significantly correlated with decreased oral function and the risk of dysphagia, supporting the notion that aging is related to deteriorating oral function in older people. Furthermore, approximately $50 \%$ of older people exhibited a DRACE score of $\geq 4$ and $15 \%$ of participants experienced choking during a meal, whether frequent or not. These results indicate that many independent community-dwelling older people have

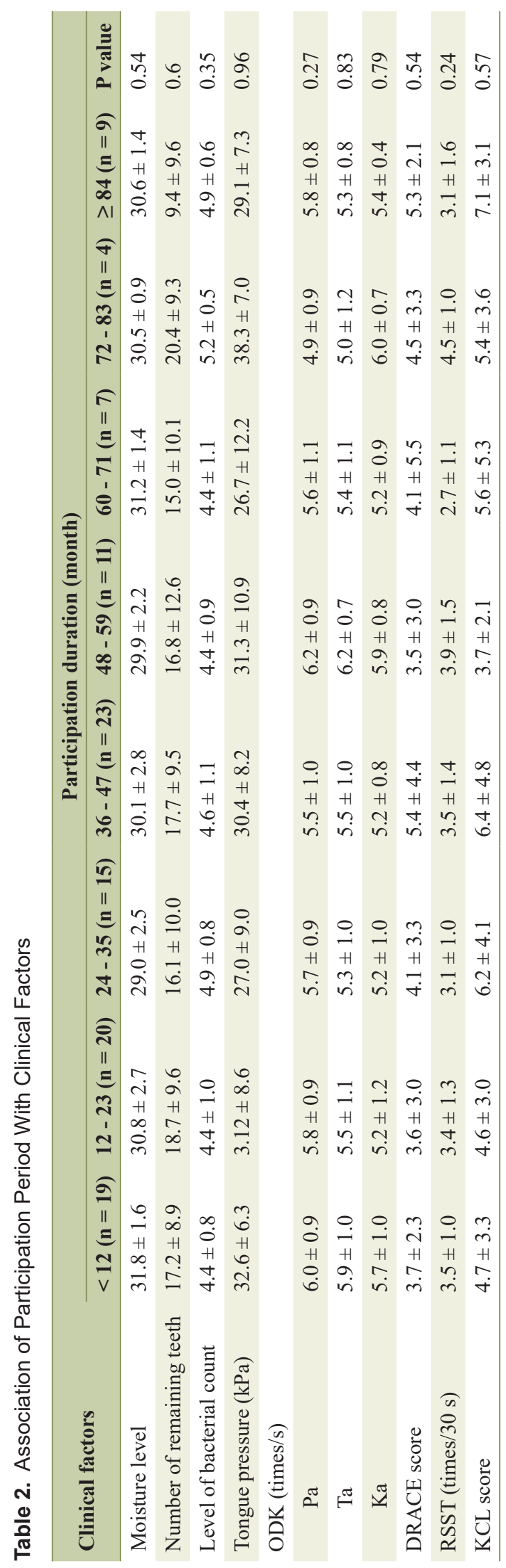


Table 3. Clinical Factors of $<3$ Years Participation and $\geq 3$ Years Participation Group

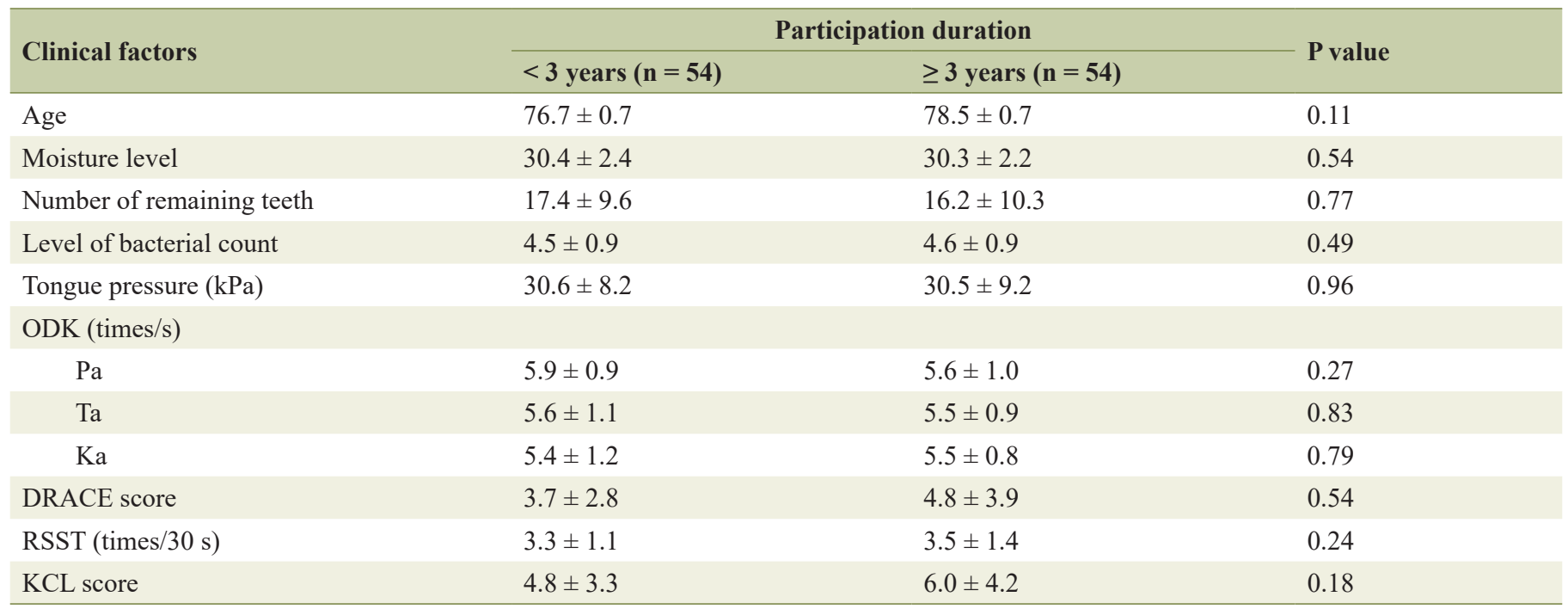

the potential risk of dysphagia. Therefore, oral rehabilitation as part of a community-based social activity is considered essential for older people to maintain their oral function. A previous study showed that oral exercise over a 3-month period prompted both maintenance and improvement of oral function, such as ODK and salivary flow, in both healthy older people and those with a high risk of oral deterioration [15]. In addition, Yamada et al [16] reported that community-dwelling older people who participated in self-managed exercise exhibited a lower incidence of requiring long-term care services than non-participants. Self-managed group exercise may be effective to maintain physical function in older people [16]. These results revealed that a regular exercise program may be useful for community-dwelling older people (i.e. people who do not need long-term care) to prevent the deterioration of physical function. Moreover, participation in health programs may play a significant role in maintaining mental health as well as physical function by promoting social activity with other people.

In the present study, we found a significant association be- tween frailty risk and decreased oral function (i.e. RSST and ODK). Decreased bite force is also reported to be significantly associated with frailty in community-dwelling older people [14]. These results suggest that deterioration of oral function is related to frailty in older people. In addition, frailty and malnutrition are closely related and are both highly prevalent in the older population [17]. Moreover, a significant association with dysphagia and malnutrition has been found in community-dwelling older people [17]. These results suggest that maintenance of swallowing ability is necessary to prevent malnutrition and dysphagia-related disease such as pneumonia in older people. Collectively, oral exercise would be helpful to maintain swallowing ability and prevent frailty in older people.

Although the percentage of frail participants was higher in the $\geq 3$ years participation group $(44.4 \%)$ when compared with the $<3$ years participation group $(27.8 \%)$, the long-term participation group exhibited a weaker negative relationship between their oral function and age than the short-term participation group. Importantly, age-dependent dry mouth was

Table 4. Correlation Between Age and Clinical Factors in $<3$ Years Participation and $\geq 3$ Years Participation Group

\begin{tabular}{lll}
\multirow{2}{*}{ Clinical factors } & \multicolumn{1}{c}{ Correlation coefficient } \\
\cline { 2 - 3 } & $<\mathbf{3}$ years $(\mathbf{n}=\mathbf{5 4})$ & $\mathbf{3}$ years $(\mathbf{n}=\mathbf{5 4})$ \\
\hline Moisture level & -0.17 & 0.19 \\
Number of remaining teeth & 0.05 & $-0.63^{* *}$ \\
Tongue pressure $(\mathrm{kPa})$ & $-0.41^{* *}$ & $-0.29^{*}$ \\
ODK (times/s) & & -0.06 \\
$\quad$ Pa & $-0.31^{*}$ & -0.11 \\
Ta & $-0.30^{*}$ & -0.25 \\
Ka & -0.24 & 0.34 \\
DRACE score & 0.12 & $-0.28^{*}$ \\
RSST (times/30 s) & $-0.42^{* *}$ & \\
\hline
\end{tabular}

${ }^{*} \mathrm{P}<0.05$, and ${ }^{* *} \mathrm{P}<0.01$. 
not found in the $\geq 3$ years participation group, suggesting that salivary gland function may not have been impaired in an age-dependent manner in this group. Our results raise the possibility that long-term participation in physical exercise may suppress the deterioration of oral function in communitydwelling older people. Long-term (i.e. 4 years) physical exercise has significantly reduced the number of people requiring long-term care services [16]. Importantly, these results suggest that long-term participation in physical exercise, including oral exercise, may contribute to the maintenance of oral function in older people.

In conclusion, long-term participation in exercise programs may suppress the deterioration of oral function in older people. There were few community salons that had been open for more than 5 years in this pilot study. Accordingly, further additional study will be necessary to clarify the significant correlation found between oral function and participation in community-based social activities, such as oral exercise programs.

\section{Competing Interest}

The authors declare that they have no competing interest.

\section{Financial Support}

This study was financially supported by university grants from Hiroshima University.

\section{References}

1. Japan Ministry of Health, Labor and Welfare. Long-Term Care Insurance System of Japan. Available at: http:// www.mhlw.go.jp/english/policy/care-welfare/care-welfare-elderly/dl/ltcisj_e.pdf.

2. Fukutomi E, Kimura Y, Wada T, Okumiya K, Matsubayashi K. Long-term care prevention project in Japan. Lancet. 2013;381(9861):116.

3. Ohara Y, Yoshida N, Kono Y, Hirano H, Yoshida H, Mataki S, Sugimoto K. Effectiveness of an oral health educational program on community-dwelling older people with xerostomia. Geriatr Gerontol Int. 2015;15(4):481-489.

4. Sakayori T, Maki Y, Hirata S, Okada M, Ishii T. Evaluation of a Japanese "Prevention of long-term care" project for the improvement in oral function in the high-risk elderly. Geriatr Gerontol Int. 2013;13(2):451-457.

5. Kaneko M, Yoshihara A, Ito K, Takano N, Fujiyama Y, Miyazaki H. The Effect of an Oral Health Care Program for Improving Oral Function in Community-dwelling El- derly. J Dent Hlth. 2009;59(1):26-33.

6. Japanese Society of Dysphagia Rehabilitation. Kunrenhou no matome. The Japanese Journal of Dysphagia Rehabilitation. 2014;18(1):55-89.

7. Kikutani T, Tamura F, Tashiro H, Yoshida M, Konishi K, Hamada R. Relationship between oral bacteria count and pneumonia onset in elderly nursing home residents. Geriatr Gerontol Int. 2015;15(4):417-421.

8. Fukushima Y, Yoda T, Araki R, Sakai T, Toya S, Ito K, Funayama $\mathrm{S}$ et al. Evaluation of oral wetness using an improved moisture-checking device for the diagnosis of dry mouth. Oral Sci Int. 2017;14(2):33-36.

9. Tsuga K, Maruyama M, Yoshikawa M, Yoshida M, Akagawa Y. Manometric evaluation of oral function with a hand-held balloon probe. J Oral Rehabil. 2011;38(9):680685 .

10. Miura H, Kariyasu M, Yamasaki K, Arai Y. Evaluation of chewing and swallowing disorders among frail community-dwelling elderly individuals. J Oral Rehabil. 2007;34(6):422-427.

11. Sewo Sampaio PY, Sampaio RA, Yamada M, Arai H. Systematic review of the Kihon Checklist: Is it a reliable assessment of frailty? Geriatr Gerontol Int. 2016;16(8):893902.

12. Yamada Y, Nanri H, Watanabe Y, Yoshida T, Yokoyama K, Itoi A, Date $\mathrm{H}$ et al. Prevalence of Frailty Assessed by Fried and Kihon Checklist Indexes in a Prospective Cohort Study: Design and Demographics of the KyotoKameoka Longitudinal Study. J Am Med Dir Assoc. 2017;18(8):733.e7-733.e15.

13. Utanohara Y, Hayashi R, Yoshikawa M, Yoshida M, Tsuga K, Akagawa Y. Standard values of maximum tongue pressure taken using newly developed disposable tongue pressure measurement device. Dysphagia. 2008;23(3):286-290.

14. Watanabe Y, Hirano H, Arai H, Morishita S, Ohara Y, Edahiro A, Murakami M, et al. Relationship between frailty and oral function in community-dwelling elderly adults. J Am Geriatr Soc. 2017;65(1):66-76.

15. Sugiyama T, Ohkubo M, Honda Y, Tasaka A, Nagasawa K, Ishida R, Sakurai K. Effect of swallowing exercises in independent elderly. Bull Tokyo Dent Coll. 2013;54(2):109-115.

16. Yamada M, Arai H. Self-management group exercise extends healthy life expectancy in frail communitydwelling older adults. Int J Environ Res Public Health. 2017;14(5):531.

17. Takeuchi K, Aida J, Ito K, Furuta M, Yamashita Y, Osaka K. Nutritional status and dysphagia risk among community-dwelling frail older adults. J Nutr Health Aging. 2014;18(4):352-357. 